\title{
Iatrogenic Hip Instability Is a Devastating Complication After the Modified Dunn Procedure for Severe Slipped Capital Femoral Epiphysis
}

\author{
Vidyadhar V. Upasani, Oliver Birke, Kevin E. Klingele, \\ Michael B. Millis, International SCFE Study Group
}

Published online: 20 September 2016

(C) The Association of Bone and Joint Surgeons( 2016

\begin{abstract}
Background The modified Dunn procedure facilitates femoral capital realignment for slipped capital femoral epiphysis (SCFE) through a surgical hip dislocation approach. Iatrogenic postoperative hip instability after this procedure has not been studied previously; however, we
\end{abstract}

Members of the International SCFE Study Group: V. V. Upasani, Rady Children's Hospital San Diego, San Diego, CA, USA; O. Birke, D. G. Little, The Children's Hospital at Westmead, Westmead, Australia; J. J. Gugenheim, Texas Orthopedic Hospital, Houston, TX, USA; P. Castañeda, Shriners Hospital for Children, Ciudad de Mexico, Mexico; J. Czubak, Samodzielny Publiczny Szpital Kliniczny, Otwock, Poland; I. Zaltz, Oakland Orthopaedic Surgeons, Royal Oak, MI, USA; K. E. Klingele, Nationwide Children's Hospital, Columbus, OH, USA; K. Mulpuri, British Columbia Children's Hospital, Vancouver, British Columbia, Canada; M. B. Millis, Boston Children's Hospital, Boston, MA, USA.

Each author certifies that he or she, or a member of his or her immediate family, has no funding or commercial associations (eg, consultancies, stock ownership, equity interest, patent/licensing arrangements, etc) that might pose a conflict of interest in connection with the submitted article.

All ICMJE Conflict of Interest Forms for authors and Clinical

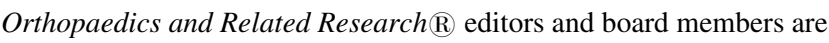
on file with the publication and can be viewed on request.

Each author certifies that his or her institution approved the human protocol for this investigation, that all investigations were conducted in conformity with ethical principles of research, and that informed consent for participation in the study was obtained.

This work was performed at Boston Children's Hospital, Boston, MA, USA.

V. V. Upasani ( $\square)$

Rady Children's Hospital San Diego, University of California

San Diego, 3030 Children's Way, Suite 410, San Diego,

CA 92123, USA

e-mail: vupasani@rchsd.org

O. Birke

The Children's Hospital at Westmead, Westmead, Australia were concerned when we observed several instances of this serious complication, and we wished to study it further. Questions/purposes The purpose of this study was to evaluate the frequency, timing, and clinical presentation (including complications) associated with iatrogenic instability after the modified Dunn procedure for SCFE.

Methods Between 2007 and 2014, eight international institutions performed the modified Dunn procedure through a surgical dislocation approach in 406 patients. During the period in question, indications varied at those sites, but the procedure was used only in a minority of their patients treated surgically for SCFE (31\% [406 of 1331]) with the majority treated with in situ fixation. It generally was performed for patients with severe deformity with a slip angle greater than $40^{\circ}$. Institutional databases were searched for all patients with SCFE who developed postoperative hip instability defined as hip subluxation or dislocation of the involved hip during the postoperative period. We reviewed in detail the clinical notes and operative records of those who presented with instability. We obtained demographic information, time from slip to surgery, type of fixation, operative details, and clinical course including the incidence of complications. Followup on those patients with instability was at a mean of 2 years (range, 1-5 years) after the index procedure. Complications were graded according to the modified Dindo-Clavien classification. Radiographic images were reviewed to

\section{K. E. Klingele}

Nationwide Children's Hospital, Columbus, OH, USA

M. B. Millis

Boston Children's Hospital, Boston, MA, USA 
measure the preoperative slip angle and the presence of osteonecrosis.

Results A total of $4 \%$ of patients treated with the modified Dunn procedure developed postoperative hip instability (17 of 406). Mean age of the patients was 13 years (range, $9-16$ years). Instability presented as persistent hip pain in the postoperative period or was incidentally identified radiographically during the postoperative visit and occurred at a median of 3 weeks (range, 1 day to 2 months) after the modified Dunn procedure. Eight patients underwent revision surgery to address the postoperative instability. Fourteen of 17 patients developed femoral head avascular necrosis and three of 17 patients underwent THA during this short-term followup.

Conclusions Anterolateral hip instability after the modified Dunn procedure for severe, chronic SCFE is an uncommon yet potentially devastating complication. Future studies might evaluate the effectiveness of maintaining anterior hip precautions for several weeks postoperatively in an abduction brace or broomstick cast to prevent this complication.

Level of Evidence Level IV, therapeutic study.

\section{Introduction}

The surgical hip dislocation technique described by Ganz et al. [4] in 2001 facilitates circumferential exposure of the femoral head and acetabulum. This approach was developed to minimize the risk of femoral head avascular necrosis after studying the complex vasculature of the medial femoral circumflex artery [5]. Over the past decade this approach has been used with increasing frequency to perform labral repair, resection of the acetabular rim, femoral head-neck osteochondroplasty, relative femoral neck lengthening, trochanteric advancement, fracture reduction, proximal femoral osteotomies, and tumor resection [10]. A number of minor complications associated with this procedure were originally described with formation of heterotopic ossification being the most common [4].

The modified Dunn procedure was described in 2007 [6] in an attempt to improve the safety of Dunn's subcapital realignment procedure for slipped capital femoral epiphysis (SCFE) [3]. The authors proposed that by combining the subcapital realignment with a surgical hip dislocation, an extended retinacular soft tissue flap could be used to preserve epiphyseal perfusion. They recommended this approach for both stable and unstable slips. Although the first case series published on 40 patients treated with the modified Dunn procedure [17] from two institutions reported only minor complications associated with this procedure including one patient with symptomatic heterotopic ossification and one patient with residual impingement who underwent an osteochondroplasty, subsequent studies have reported a higher risk of complications and more severe complications including avascular necrosis and fracture of internal fixation devices, resulting in slip progression, pain, or further revision surgical procedures $[9,11,13,16]$.

Iatrogenic hip instability after the modified Dunn procedure has received little attention [13]. In fact, the original description of the procedure recommended resection of the ligamentum teres and loose closure of the hip capsule to avoid tensioning the retinacular vessels [17]. After identifying a few instances of this complication, we felt a multicenter review would be important to inform our clinical practice. The purpose of this study was to evaluate the frequency, timing, and clinical presentation (including complications) associated with iatrogenic instability after the modified Dunn procedure for SCFE.

\section{Patients and Methods}

After obtaining institutional review board approval, a retrospective study was performed at eight institutions in North America, Europe, and Australia. Each site reviewed the records of all patients treated with the modified Dunn procedure for SCFE and identified cases of hip instability that developed in the postoperative period. All modified Dunn procedures were performed according to the technique described by Leunig et al. [6]. Intraoperative monitoring of femoral epiphyseal perfusion varied among surgeons ranging from no monitoring, to using a drill hole to identify bleeding, to use of an intraosseous monitor to determine pulse pressure. Epiphyseal fixation also varied between surgeons ranging from threaded Kirschner wires, to $4.5-\mathrm{mm}$ screws, to $6.5-\mathrm{mm}$ cannulated screws. The median number of modified Dunn procedures performed at the eight institutions was 56 (range, 1-128). The median number of total patients with SCFE treated at each center during the study period was 253 (range, 60-657).

Between January 2007 and December 2014, the eight international institutions performed the modified Dunn procedure through a surgical dislocation approach in 406 patients. During the period in question, indications varied at those sites, but the modified Dunn procedure was used only in a small minority of their patients treated surgically for SCFE (31\% [406 of 1331]), and it generally was performed for patients with severe proximal femoral deformity and a slip angle of greater than $40^{\circ}$. The majority of our patients with SCFE were treated with in situ fixation.

Clinic notes and operative records were reviewed to obtain demographic information, time from slip to surgery, type of fixation, operative details, and clinical course including the incidence of complications. Radiographic 
images were reviewed to measure the preoperative slip angle and the presence of osteonecrosis. Followup on the patients with instability was at a mean of 2 years (range, 1-5 years) after the index procedure (modified Dunn).

Complications were graded according to the adapted Dindo-Clavien classification $[1,2]$ by an independent assessor (VVU) who was not involved in the clinical care of these patients. Patients with Grade I complications underwent no treatment and had no clinical relevance with no deviation from routine followup. Patients with Grade II complications resulted in deviation from the normal postoperative course and outpatient treatment, either pharmacologic or close monitoring. Grade III complications resulted in surgical or radiologic interventions or an unplanned hospital admission. Grade IV complications were life-threatening or had the potential for permanent disability. Death was considered a Grade V complication.

\section{Results}

A total of $4 \%$ of patients treated with the modified Dunn procedure developed postoperative hip instability (17 of 406). Ten of the patients were male, and seven were female (Table 1). The mean age of the patients was 13 years (range, 9-16 years).
Instability presented as persistent hip pain in the postoperative period or as an incidental radiographic finding at the postoperative visit and was identified at a median of 3 weeks (range, 1 day to 2 months) after the modified Dunn procedure. This complication was diagnosed radiographically with subluxation or dislocation of the femoral head from the acetabulum.

Eight patients underwent revision surgery to address the postoperative dislocation including open reduction and capsulorrhaphy $(n=6)$, application of a hinged external fixator $(\mathrm{n}=1)$ (Fig. 1), and greater trochanteric transfer $(n=1)$. Fourteen patients in this series had more than one complication. There were 17 Grade III complications and 13 Grade IV complications. Postoperative instability was considered a Grade III complication (Fig. 2). Nine patients were treated with closed reduction and casting $(\mathrm{n}=6)$, abduction bracing $(n=2)$, or balanced suspension $(n=1)$. Fourteen patients developed avascular necrosis with severe degenerative joint disease and femoral head collapse. In the short-term followup period, three patients were revised with THA, two patients were treated with intertrochanteric femoral osteotomy, one patient was treated with epiphyseal drilling, one patient was treated with a vascularized fibula graft, and eight patients underwent removal or revision of implants attributable to joint penetration.

Table 1. Patient demographics and slipped capital femoral epiphysis classification

\begin{tabular}{|c|c|c|c|c|c|c|c|c|c|}
\hline $\begin{array}{l}\text { Patient } \\
\text { number }\end{array}$ & $\begin{array}{l}\text { Date of } \\
\text { birth }\end{array}$ & Sex & $\begin{array}{l}\text { Age } \\
\text { (years) }\end{array}$ & Side & $\begin{array}{l}\text { Date of } \\
\text { surgery }\end{array}$ & $\begin{array}{l}\text { Last clinic } \\
\text { visit }\end{array}$ & $\begin{array}{l}\text { Followup } \\
\text { (months) }\end{array}$ & $\begin{array}{l}\text { Loder } \\
\text { classification } \\
{[7]}\end{array}$ & Chronicity \\
\hline 1 & $5 / 31 / 99$ & $\mathrm{M}$ & 13 & $\mathrm{~L}$ & $8 / 23 / 12$ & $9 / 18 / 14$ & 25 & Stable & Chronic \\
\hline 2 & $1 / 30 / 96$ & M & 11 & $\mathrm{R}$ & 7/27/07 & $3 / 2 / 12$ & 55 & Unstable & Acute on chronic \\
\hline 3 & $7 / 27 / 96$ & M & 12 & $\mathrm{R}$ & 2/10/09 & $11 / 19 / 10$ & 21 & Unstable & Acute on chronic \\
\hline 4 & $2 / 7 / 99$ & M & 12 & $\mathrm{R}$ & $8 / 24 / 11$ & $5 / 16 / 13$ & 21 & Stable & Chronic \\
\hline 5 & $11 / 24 / 98$ & $\mathrm{~F}$ & 13 & $\mathrm{R}$ & $2 / 17 / 12$ & $3 / 19 / 13$ & 13 & Unstable & Acute on chronic \\
\hline 6 & $5 / 18 / 98$ & M & 12 & $\mathrm{R}$ & $7 / 2 / 10$ & 7/31/12 & 25 & Stable & Acute on chronic \\
\hline 7 & $5 / 2 / 00$ & $\mathrm{~F}$ & 13 & $\mathrm{R}$ & $9 / 9 / 13$ & $3 / 17 / 16$ & 30 & Stable & Acute on chronic \\
\hline 8 & $12 / 21 / 93$ & $\mathrm{M}$ & 16 & $\mathrm{R}$ & 12/7/09 & $11 / 3 / 11$ & 23 & Stable & Chronic \\
\hline 9 & $3 / 31 / 00$ & $\mathrm{~F}$ & 13 & $\mathrm{R}$ & 9/30/13 & $12 / 2 / 14$ & 14 & Stable & Chronic \\
\hline 10 & 6/21/99 & M & 13 & $\mathrm{~L}$ & $4 / 26 / 13$ & $3 / 24 / 15$ & 23 & Unstable & Acute on chronic \\
\hline 11 & 2/10/98 & $\mathrm{F}$ & 13 & $\mathrm{R}$ & 7/27/11 & $10 / 29 / 12$ & 15 & Unstable & Acute on chronic \\
\hline 12 & $10 / 7 / 95$ & M & 15 & $\mathrm{R}$ & $4 / 8 / 11$ & 2/18/13 & 23 & Stable & Chronic \\
\hline 13 & 8/18/99 & $\mathrm{M}$ & 14 & $\mathrm{~L}$ & $4 / 2 / 14$ & 1/18/16 & 26 & Stable & Chronic \\
\hline 14 & $3 / 12 / 02$ & $\mathrm{~F}$ & 10 & $\mathrm{~L}$ & $11 / 6 / 12$ & $3 / 23 / 15$ & 30 & Stable & Chronic \\
\hline 15 & $11 / 24 / 03$ & $\mathrm{~F}$ & 9 & $\mathrm{~L}$ & $10 / 22 / 13$ & $25 / 1 / 2016$ & 27 & Unstable & Acute on chronic \\
\hline 16 & $11 / 12 / 02$ & $\mathrm{~F}$ & 10 & $\mathrm{~L}$ & $11 / 27 / 12$ & $11 / 4 / 2016$ & 40 & Stable & Acute on chronic \\
\hline 17 & $12 / 22 / 00$ & M & 13 & $\mathrm{~L}$ & $10 / 7 / 14$ & 7/3/2016 & 17 & Unstable & Acute on chronic \\
\hline
\end{tabular}

$\mathrm{M}=$ male; $\mathrm{F}=$ female; $\mathrm{L}=$ left; $\mathrm{R}=$ right. 
Fig. 1A-G (A) AP and (B) frog lateral radiographs show a 13-year-old boy who presented with 6 months of posterolateral hip pain aggravated by activity. $\mathrm{He}$ was able to ambulate without assistive devices with a mild antalgic limp. He was diagnosed with a severe, chronic, stable left SCFE and mild right slip deformity. He underwent in situ pin fixation of the right hip and a modified Dunn procedure for the left. (C) AP radiograph at 6 weeks postoperatively demonstrates severe subluxation of the left hip with no clear evidence of avascular necrosis. (D) AP radiograph after application of an articulated hip external fixator. (E) AP radiograph after sequential 1-mm distraction daily until the femoral head was at the level of the acetabulum. Nearly 4 weeks after application of the distractor, he returned to the operating room to remove the external fixator. An arthrogram was performed to check reduction and stability followed by a limited anterior surgical approach to directly visualize the reduction. A static external fixator was then used to keep the hip concentrically reduced and the patient was maintained nonweightbearing. (F) AP and (G) frog lateral radiographs 2 years after surgery demonstrate femoral head collapse resulting from avascular necrosis with joint space narrowing. He had a severe left-sided Trendelenburg gait and ambulated with a cane.
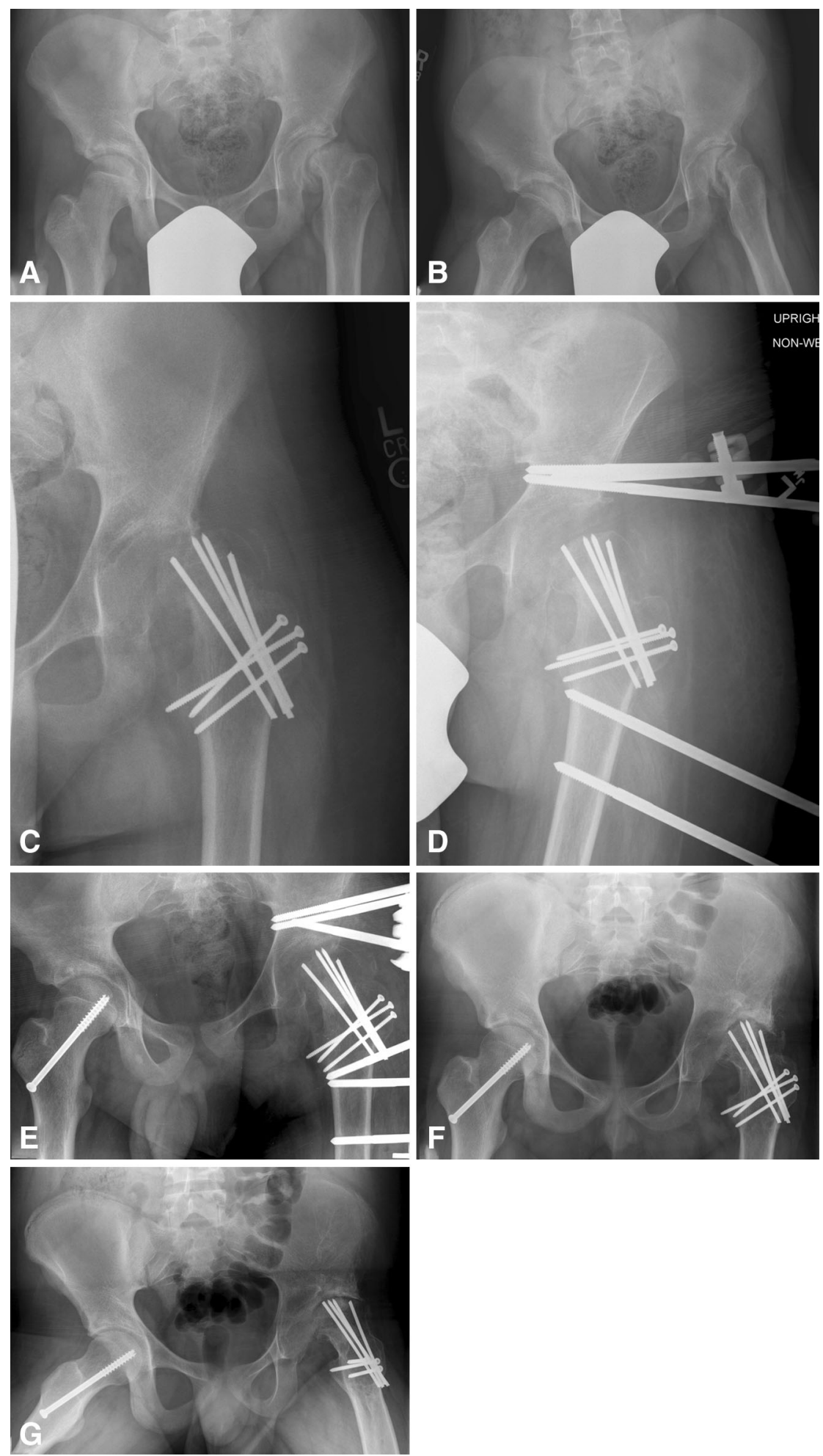

\section{Discussion}

Postoperative iatrogenic hip instability (leading to subluxation or dislocation) is a devastating complication of the modified Dunn procedure. To this point, this complication has received little attention [13]; however, after identifying a few sporadic instances of this complication, we performed a multicenter review, which identified 17 cases from eight institutions over a 7-year period. All these patients had severe proximal femoral deformity with a slip 
Fig. 2A-H (A) AP and (B) lateral radiographs show a 12year-old boy who presented to the emergency department with worsening right hip pain and limp. He had been experiencing right knee and hip pain for more than 1 year. He was diagnosed with a moderate, chronic, stable SCFE. (C) AP and (D) frog lateral radiographs after in situ pin fixation. (E) AP radiograph after the modified Dunn procedure was performed 4 months after the index procedure. (F) AP radiograph at the 2-week postoperative visit demonstrates high dislocation. This was treated with open reduction and 6 weeks of abduction bracing. (G) AP and (H) lateral radiographs 2 years after the modified Dunn procedure. There is radiographic evidence of mild lateral femoral head avascular necrosis; however, the patient has no hip pain. He has a reciprocal heel-toe gait with approximately $10^{\circ}$ externally rotated foot progression angle. Hip flexion is comfortable to $100^{\circ}$. He has $20^{\circ}$ of internal rotation and $40^{\circ}$ of external rotation symmetrically.
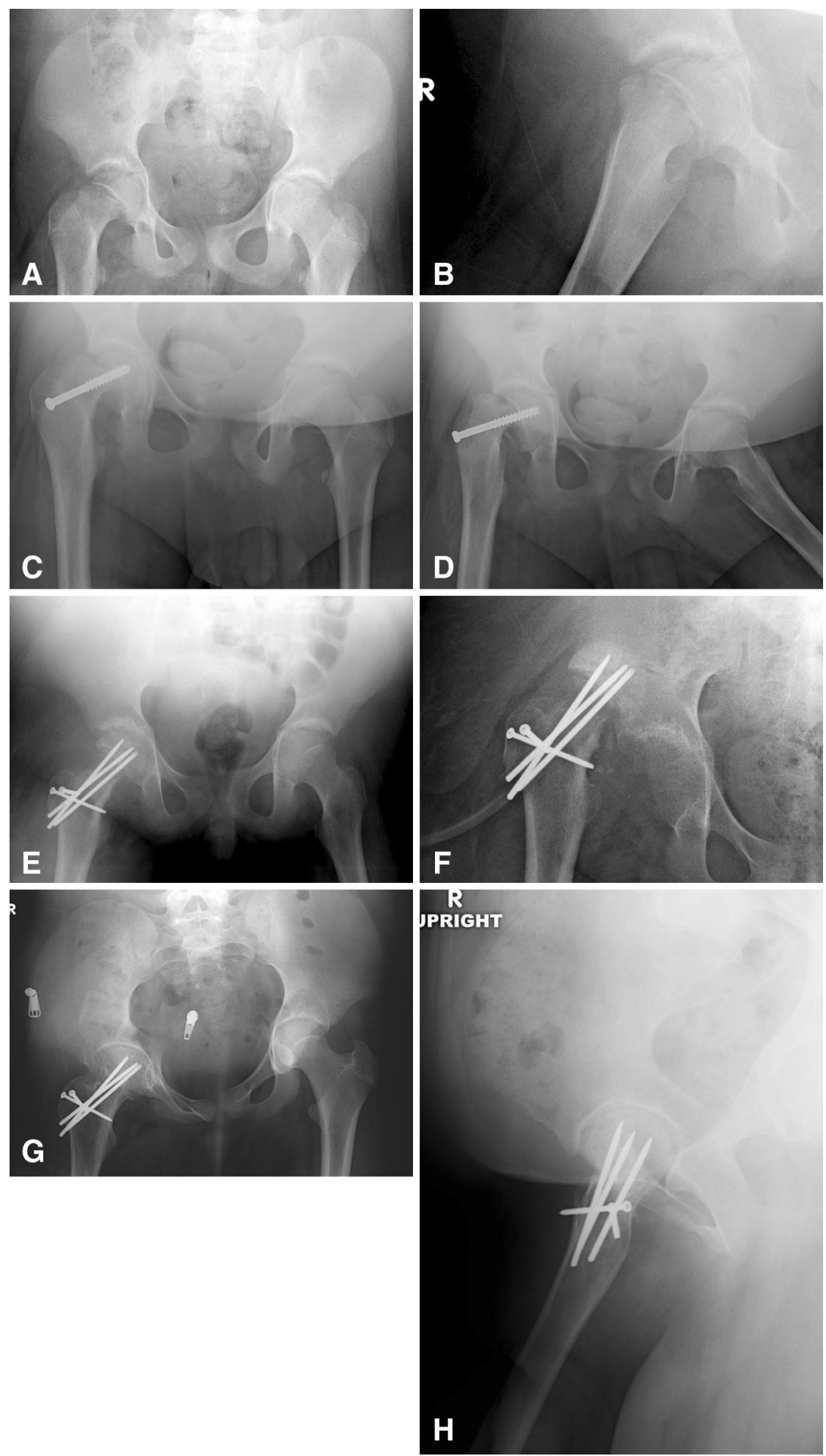

angle of greater than $40^{\circ}$ and chronic or acute-on-chronic SCFEs based on the Loder classification [7]. Nearly half of these patients underwent revision surgery and a majority developed femoral head avascular necrosis.
This study has a number of limitations. First, there is clear selection bias because only a minority of all patients with SCFE treated at the eight institutions underwent the modified Dunn procedure, whereas the vast majority was 
treated with in situ fixation. It is possible that the postoperative instability is a function of slip severity as much (or more) as of the procedure itself; certainly it seems less common in less severe slips. Additionally, we do not have complete followup on the entire group of patients treated for SCFE with the Dunn procedure, and so our reported frequency of iatrogenic instability must therefore be considered a conservative estimate. Similarly, because our followup in this group of patients who experienced postoperative instability is short, the morbidity observed may be underestimated. Additionally, because some of the patients included in this analysis had previous in situ stabilization before the modified Dunn procedure, it is possible that the instability complication was the result of performing these two procedures in series. Also, it can be difficult in some patients to distinguish between chondrolysis and avascular necrosis; in this series, we recorded all questionable instances as avascular necrosis, although it is possible that some patients might instead have had chondrolysis. Both diagnoses are considered major complications, and both generally progress to severe degenerative joint disease. Finally, subjective scores or functional assessments were not performed on these patients.

We observed iatrogenic hip instability in $4 \%$ of patients who underwent the modified Dunn procedure resulting in severe morbidity in this subset of patients. The patients in this series all had severe deformity with a slip angle of greater than $40^{\circ}$ and chronic or acute-on-chronic disease. It is difficult to directly compare outcomes between different surgical approaches because most previous publications combine patients with a variety of disease severities (based on slip angle, chronicity, and epiphyseal stability). However, looking at a comparably complex series of patients with SCFE, Vanhegan et al. reported on 57 patients treated with a subcapital osteotomy for severe SCFE (slip angle greater than $50^{\circ}$ ) with a $22 \%$ overall complication rate and $10.5 \%$ rate of avascular necrosis [14]. Long-term results of the Imhauser intertrochanteric osteotomy for severe SCFE (slip angle greater than 40) were presented by Trisolino et al. [12]. Their study reported a cumulative 39 years survivorship free from THA of $68.5 \%$ with four early postoperative complications in 47 hips. Similarly, Parsch et al. reported on 19 patients with severe, chronic SCFE who were treated with an intertrochanteric corrective osteotomy as a subset of their 299 total patients with SCFE with only one case of postoperative avascular necrosis [8]. On the other hand, Wensaas et al. presented long-term outcomes on 66 patients with SCFE and reported that six of their eight patients with a severe chronic slip (mean slip angle of $53^{\circ}$ ) had poor clinical outcomes after bone-peg epiphysiodesis and corrective femoral osteotomy [15].
Compared with other series of comparably challenging SCFEs, we found postoperative instability tended to occur earlier (median of 3 weeks) after the modified Dunn procedure. The most severe complications from alternate surgical treatments for severe, chronic SCFE (such as avascular necrosis and chondrolysis) typically appear 3 to 15 months after the index procedure $[9,12,15,16]$. In contrast, hip subluxation or dislocation was observed as early as the first postoperative day. The dislocation that was identified 2 months postoperatively was in a patient who was noncompliant with postoperative instructions and did not follow up at their scheduled appointment. It is possible that increased vigilance in the early postoperative period with strict anterior hip precautions or with postoperative immobilization (like with a broomstick cast) that limits hip extension and external rotation may decrease the incidence of this complication.

The sequelae of the complications we observed were in many patients quite severe. This finding is also different from most reports on comparably challenging SCFE presentations $[9,14,16]$. The majority of patients in this series developed degenerative joint disease and three went on to undergo THA. It is important to note that this subset represents only $4 \%$ of the total number of modified Dunn procedures performed; however, because the sequelae are so problematic, these results may dissuade other centers from using this approach. The eight centers involved in this study had varying experience with the modified Dunn procedure, and there was a large range in the volume of total patients with SCFE treated at each site. However, because the number of postoperative instability cases was so few, ranging from one to three per center, it is difficult to state whether surgeon experience or a learning curve played a role in this complication.

Although we cannot know why hip instability was so common with this approach in this series, we speculate that it was a combination of the severity of the SCFEs treated here and elements specific to the procedure. These elements might have included the dislocation of the hip to perform the capital realignment; extension-external rotation contractures that may have developed over months in some of the patients with more chronic SCFEs; and attenuated or incompetent anterior hip capsular tissue (again, in patients with chronic SCFE), perhaps further weakened by the procedure's extensive capsulotomy and loose repair, which must seek to avoid constriction of the posterior retinaculum and vessels. Finally, if excessive shortening of the femoral neck is performed, this too might contribute to postoperative instability. Regardless of the cause, the combination of more severe SCFE and the modified Dunn procedure in this series was associated with devastating complications in $4 \%$ (17 of 406) of our patients. 
Anterolateral hip instability after the modified Dunn procedure in patients with severe and/or chronic SCFE is an uncommon yet potentially devastating complication. This study confirms that there may be substantial morbidity associated with this complex procedure. It is unclear from our case series if this complication could be avoided in patients with more severe SCFE or whether an alternate, more conservative approach would be preferable. Future studies will need to determine if distalization of the greater trochanter to achieve relative femoral neck lengthening, maintaining anterior hip precautions, or using postoperative broomstick immobilization for several weeks may avoid the postoperative instability described in this case series.

Acknowledgments We recognize Rasika Deshpande BS, and Drs Kevin Horowitz, Michal Lenkiewicz, and Alfred Mansour III for their assistance in data collection and manuscript preparation.

\section{References}

1. Clavien PA, Strasberg SM. Severity grading of surgical complications. Ann Surg. 2009;250:197-198.

2. Dindo D, Demartines N, Clavien PA. Classification of surgical complications: a new proposal with evaluation in a cohort of 6336 patients and results of a survey. Ann Surg. 2004;240:205213.

3. Dunn DM. The treatment of adolescent slipping of the upper femoral epiphysis. J Bone Joint Surg Br. 1964;46:621-629.

4. Ganz R, Gill TJ, Gautier E, Ganz K, Krugel N, Berlemann U. Surgical dislocation of the adult hip a technique with full access to the femoral head and acetabulum without the risk of avascular necrosis. J Bone Joint Surg Br. 2001;83:1119-1124.

5. Gautier E, Ganz K, Krugel N, Gill T, Ganz R. Anatomy of the medial femoral circumflex artery and its surgical implications. $J$ Bone Joint Surg Br. 2000;82:679-683.

6. Leunig M, Slongo T, Kleinschmidt M, Ganz R. Subcapital correction osteotomy in slipped capital femoral epiphysis by means of surgical hip dislocation. Oper Orthop Traumatol. 2007;19:389-410.

7. Loder RT, Richards BS, Shapiro PS, Reznick LR, Aronson DD. Acute slipped capital femoral epiphysis: the importance of physeal stability. J Bone Joint Surg Am. 1993;75:1134-1140.

8. Parsch K, Zehender H, Buhl T, Weller S. Intertrochanteric corrective osteotomy for moderate and severe chronic slipped capital femoral epiphysis. J Pediatr Orthop B. 1999;8:223-230.

9. Sankar WN, Vanderhave KL, Matheney T, Herrera-Soto JA, Karlen JW. The modified Dunn procedure for unstable slipped capital femoral epiphysis: a multicenter perspective. J Bone Joint Surg Am. 2013;95:585-591.

10. Sink EL, Beaule PE, Sucato D, Kim YJ, Millis MB, Dayton M, Trousdale RT, Sierra RJ, Zaltz I, Schoenecker P, Monreal A, Clohisy J. Multicenter study of complications following surgical dislocation of the hip. J Bone Joint Surg Am. 2011;93:11321136.

11. Tibor LM, Sink EL. Risks and benefits of the modified Dunn approach for treatment of moderate or severe slipped capital femoral epiphysis. J Pediatr Orthop. 2013;33(Suppl 1):S99102.

12. Trisolino G, Pagliazzi G, Di Gennaro GL, Stilli S. Long-term Results of combined epiphysiodesis and imhauser intertrochanteric osteotomy in SCFE: a retrospective study on 53 hips. J Pediatr Orthop. 2015 Nov 18. [Epub ahead of print].

13. Upasani VV, Matheney TH, Spencer SA, Kim YJ, Millis MB, Kasser JR. Complications after modified Dunn osteotomy for the treatment of adolescent slipped capital femoral epiphysis. $J$ Pediatr Orthop. 2014;34:661-667.

14. Vanhegan IS, Cashman JP, Buddhdev P, Hashemi-Nejad A. Outcomes following subcapital osteotomy for severe slipped upper femoral epiphysis. Bone Joint J. 2015;97B:17181725 .

15. Wensaas A, Svenningsen S, Terjesen T. Long-term outcome of slipped capital femoral epiphysis: a 38-year follow-up of 66 patients. J Child Orthop. 2011;5:75-82.

16. Zaltz I, Baca G, Clohisy JC. Unstable SCFE: review of treatment modalities and prevalence of osteonecrosis. Clin Orthop Relat Res. 2013;471:2192-2198.

17. Ziebarth K, Zilkens C, Spencer S, Leunig M, Ganz R, Kim YJ. Capital realignment for moderate and severe SCFE using a modified Dunn procedure. Clin Orthop Relat Res. 2009;467:704 716. 